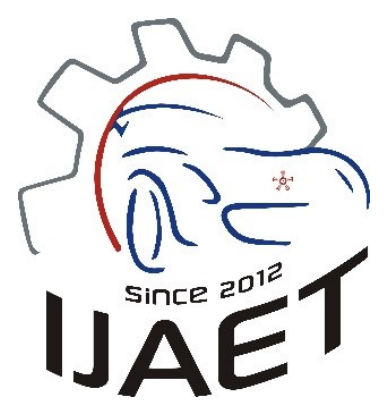

e-ISSN: 2146 - 9067

International Journal of Automotive

Engineering and Technologies

journal homepage:

https://dergipark.org.tr/en/pub/ijaet

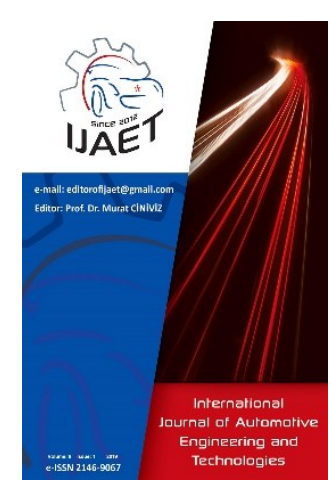

Original Research Article

\title{
Experimental study on effects of vehicle speed variations on effective rolling radius according to the changes in tire pressure
}

\section{Hakan Köylü ${ }^{\text {* }}$ \\ 1, * Department of Automotive Engineering, Faculty of Technology, Kocaeli University, Turkey \\ ARTICLE INFO \\ ABSTRACT}

$10000-0002-3548-0484$

* Corresponding author hkoylu@kocaeli.edu.tr

Received: Sep 10, 2019

Accepted: June 7, 2020

Published by Editorial Board Members of IJAET

(C) This article is distributed by Turk Journal Park System under the CC 4.0 terms and conditions.
This study aims to experimentally examine the effects of vehicle speed changes on the vertical resonance frequency of effective rolling radius variations for applying to TPMS (Tire Pressure Monitoring System). For this, the road tests have been conducted at constant vehicle speeds $30,60,90 \mathrm{~km} / \mathrm{h}$ for tire pressures $15,20,25,30,35 \mathrm{psi}$. In these tests, the effective rolling radius changes have been measured. Test results show that the vehicle speed changes cause the resonance frequency to shift at same tire pressure. Therefore, this study shows that the changes in vertical resonance frequency resulting from vehicle speed changes may cause wrong tire pressure information to be taken by TPMS.

Keywords: Effective rolling radius, vehicle speed, resonance frequency, TPMS.

\section{Introduction}

In the vehicles, acceleration, braking and handling performances greatly depend on the tire-road contact patch. Because the braking and driving forces are not transferred onto road, when the contact weakens. This causes inconsistent braking maneuver, long acceleration process, unstable handling and non-uniform tire wear. When it is considered that only vehicle component which is in contact with road is the pneumatic tire, it is clear that the pneumatic tire parameters determining the changes in tire-road contact have direct effect on vehicle dynamics. One of the most important tire parameters is tire inflation pressure. Because, the pressure distribution inside tire determines the area of tire-road contact patch. In other words, if the tire pressure decreases relative to nominal pressure, the pressure distribution is centered on shoulder and the contact area decreases in the middle of tire. Also, if the tire pressure increases relative to nominal pressure, the pressure distribution is concentrated on the middle of tire contact patch and the contact area decreases in shoulder of tire [1]. When the tire pressure increases or decreases, fuel consumption and exhaust emissions increase considerably. Also, it deteriorates braking and driving performance of the vehicle. It was reported that fuel consumption increases and tire lifetime decreases by $20 \%$ for every 0.2 bar under-inflation [2]. They also expressed that tire problems are the third most common breakdown for passenger vehicles. It is stated that a malfunction of the tires in motion due to a tire puncture can cause serious accidents and endanger human life [3]. Also, low tire pressure is very dangerous for especially heavy 
loaded vehicles as it travelled in a highway at hot weather conditions; in such case, tires may blowout [4]. These can only be avoided, if the air pressure in the tires can be measured even during driving. For this reason, in 2000, the U.S. Transportation Recall Enhancement, Accountability, and Documentation Act (TREAD) requested to investigate the implementation of a pressure drop warning system on vehicles from the National Highway Transport Safety Authority (NHTSA). Beginning with 2006 models, all passenger cars and trucks in the United States are required to have tire-pressure monitoring systems (TPMSs) [5]. Also, it stated that TPMS has been mandatory for every new vehicle sold in the United States since September 2007 by [6]. Moreover, Europe is in the process of adopting similar regulations as a way to reduce carbon dioxide emissions, boost fuel economy, and enhance safety. China is also considering similar legislation.

A TPMS is a driver-assist system that warns the driver when the tire pressure decreases and increases relative to nominal tire pressure. TPMS systems are classified in two different categories. These are based on direct and indirect methods. Direct TPMS uses a pressure sensor directly mounted on the wheels or tires of a vehicle. Firstly, the pressure inside the tire is measured using a pressure transducer. Then, the pressure information is being sent to the vehicle to warn the driver of under or over inflation of a tire. The pressure information is commonly transmitted to the vehicle using radio frequency (RF) technology. Therefore, in direct TPMSs, the pressure drop is calculated based on actual pressure measurements through sensors. A direct TPMS can also inform the driver about pressure deviations as low as \pm 0.1 bar, that is, \pm 1.45 psi $[5,7]$. However, this method is very expensive due to the extra hardware requirements. Another alternative to the direct method is indirect tire pressure monitoring system. This system uses existing sensors and software algorithms. The indirect systems are very cost-effective and they have no extra hardware. An indirect TPMS predicts tire pressure drop using estimation algorithm and thus does not require tire pressure sensors. Available indirect TPMSs are generally based on wheel speed measurements. In this system, it is considered that the tire pressure decreases and the vehicle's weight causes the tire's diameter to decrease. This leads the tire to rotate at more different rate than that of full pressure [8]. However, several shortcomings are associated with indirect TPMS based on speed signals of four wheels. First, the system does not provide the actual pressure of each tire and it works only when the vehicle is in motion. Additionally, the system does not give a warning when two tires are equally underinflated on the same side or same axle. This is also valid, when all four tire pressures are equally low. It warns the driver only when the pressure drop is more than $25 \%$. At same time, this system may generate false warnings when the vehicle is moving on a curved road or during tire slip on snowy roads $[5,8]$. For this, a model-based estimator was developed to predict tyre inflation pressure via tyre vertical stiffness by using only standard wheel speed sensors during normal driving in a turn [9]. Also, some studies related to in indirect TPMS made the detection of low tire pressure feasible in all tyres at same time by analyzing vibration of each wheel during moving in a turn [10].

As a result, a number of studies are performed for indirect TPMS $[4,6,10]$. In these studies, the methods based on wheel speed through effective rolling radius signals have been used. In here, the method based on wheel speed changes has some shortcomings as mentioned in above. For this reason, this study has focused on the methods based on the changes in effective rolling radius. In these methods, it is clearly seen that the changes in vehicle speed are not considered in indirect TPMS. Whereas NHTSA has reported that the increases in vehicle speed cause longitudinal and lateral friction coefficients to decrease under both wet and dry road conditions. Then, they have explored that the decreases in tire inflation pressure lead the friction coefficient to drop 5\% and $9 \%$, respectively as the vehicle speed increases. Moreover, the effective rolling radius reduced greatly as the vehicle speed increases at same tire pressure [11]. This situation was also seen in frequency domain and it was explored that the increases in vehicle speed vary the peak amplitude of rolling radius [12]. These results show that the vehicle speed changes cause the fluctuations in effective 
rolling radius and wheel speed as shown in Eq. (1).

$$
V_{x}=\dot{\varphi} R_{e}
$$

where $V_{x}$ is vehicle speed, $\dot{\varphi}$ is angular velocity of wheel and $R_{e}$ is effective rolling radius. Therefore, the vehicle speed has a great effect on rolling radius with different tire pressures. For this, the monitoring systems considering effective rolling radius should be developed by considering the changes in vehicle speed. Thus, in this study, the effects of vehicle speed changes on resonance frequencies of effective rolling radius are investigated according to various tire inflation pressures. For this, constant speed tests are conducted at 30,60, $100 \mathrm{kph}$, respectively for $15,20,25,30$ and $35 \mathrm{psi}$ tire pressure. In tests, the effective rolling radius changes are measured. The frequency responses of the measured parameter are obtained by using FFT filtered with low pass filter. Therefore, in this study, the effects of vehicle speed on the effective rolling radius will be explored according to the changes in location of resonance frequency and the oscillation amplitudes of effective rolling radius for different vehicle speed and tire inflation pressures to employ the effective rolling radius in TPMS, irrespective of vehicle speed variations.

\section{Tire Model}

In this study, the effects of vehicle speeds on variations in effective rolling radius are investigated based on the changes in vertical resonance frequency of wheel for the different tire pressures. For this, the vertical vibration analysis of tire is carried out. In this analysis, tire is modeled as a spring in vertical direction as shown in Fig.1. The tire spring is described with vertical stiffness of tire. In this model, tire damping is neglected, since tire spring stiffness is much higher than viscous damping coefficient of tire. In Fig.1, tire is divided into belt side and rim side. Rim side is rigid, but belt side can deform in vertical direction relative to rim side as shown in Fig.2. According Fig.2, the relationship among the decreases in effective rolling radius, wheel speed and vehicle speed is described as follows [2]:

$$
\dot{\varphi}=\frac{V_{x}}{R_{e}-\delta R_{e}}
$$

where $\dot{\varphi}$ is wheel speed, $R_{e}$ is effective rolling radius, $\delta R_{e}$ is the decrease in effective rolling radius affected by deflation and $V_{x}$ is vehicle speed.

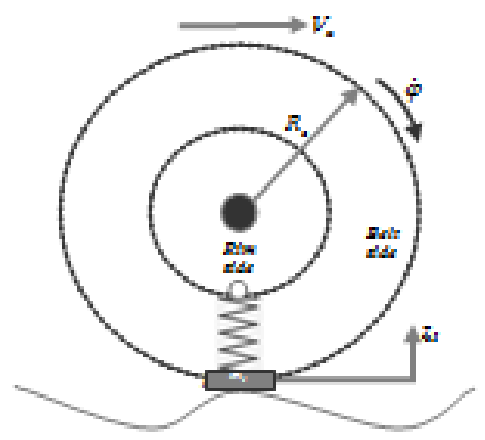

Figure 1. Tire vibration model with nominal inflation pressure

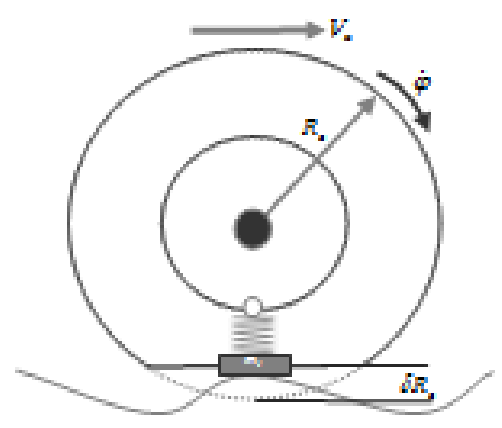

Figure 2. Tire vibration model with deflated tire

The Eq.(2a) indicates that the wheel rotates faster as the decrease in effective rolling radius increases because of the tire deflation. In here, the vehicle speed is constant. However, if the vehicle's speed changes, the following description is taken into consideration by rearranging Eq.(2a) relative to $\delta R_{e}$.

$\delta R_{e}=R_{e}-\frac{V_{x}}{\dot{\varphi}}$

As shown in Eq.(2b), the decreases in the effective rolling radius are dependent on the ratio of vehicle speed to wheel speed. Hence the increases in vehicle speed cause the effective rolling radius to decrease. Because, the increases in vehicle speed cause the friction to decrease between tire and road because of the upward moving of wheel. This shows that the vehicle speed changes can lead fluctuations in effective rolling radius changes. Also, during accelerating of the vehicle, the tire vibrates in 
the vertical direction and causes the effective rolling radius to fluctuate with a specific resonance frequency.Therefore, this frequency must be determined. For this, the relationship between effective rolling radius and vertical resonance frequency for free vibration according to Fig. (1) and the following dynamic equation is used.

$$
\begin{aligned}
& m_{1} \ddot{z}_{1}+k z_{1}=0 \\
& m_{1} \ddot{z}_{1}=-k z_{1}
\end{aligned}
$$

where $m_{1}$ is mass of belt side, $k$ is vertical tire stiffness and $z_{1}$ is the vertical displacement of $m_{1}$. The vertical displacement and acceleration of belt side mass are described as follows:

$$
\begin{aligned}
& z_{1}=z \sin \omega t \\
& \ddot{z}_{1}=-\omega^{2} z \sin \omega t
\end{aligned}
$$

When Eqs. (5a) and (5b) is substituted into Eq. (4), the following description is obtained.

$$
\begin{aligned}
& -m_{1} \omega^{2} z \sin \omega t=-k z \sin \omega t \\
& m_{1} \omega^{2}=k
\end{aligned}
$$

Therefore, the vertical resonance frequency is described as follows:

$$
\omega=\sqrt{\frac{k}{m_{1}}}
$$

The description reflecting the decreases in vertical tire stiffness is as follows:

$$
\begin{aligned}
& \omega_{r}=\sqrt{\frac{k-\Delta k}{m_{1} g}} \\
& \omega_{r}=\sqrt{\frac{k}{m_{1} g}-\frac{\Delta k}{m_{1} g}}
\end{aligned}
$$

where $\omega_{r}$ is the vertical resonance frequency of wheel and $\Delta k$ is the decrease in tire spring stiffness by deflation. Thereby, when the tire inflation pressure decreases, the springconstant is decreased, and this yields a lower resonance frequency. The most significant mode for this vibration is about 7-20 Hz. In order to occur the interaction between vertical resonance frequency and effecive rolling radius, $\Delta k$ is described as follows:

$$
\Delta k=\frac{m_{1} g}{\Delta z}
$$

where $\Delta z$ is vertical deformation of tire and it is equivalent to the decrease in effective rolling radius $\left(\delta R_{e}\right)$. For this, the effective rolling radius is described according to the vertical deformation as follows [13]:

$$
\begin{aligned}
& R_{e}=(1-\eta) \Delta z \\
& \Delta z=\frac{R_{e}}{(1-\eta)}
\end{aligned}
$$

where $\eta$ is experimental constant. It takes 0.4 and 0.1 values for cross ply and radial ply, respectively [14]. Therefore, the decrease in tire spring stiffness is described according to the decrease in effective rolling radius as follows:

$$
\begin{aligned}
& \Delta k=\frac{m_{1} g(1-\eta)}{R_{e}-\delta R_{e}} \\
& \Delta k=m_{1} g(1-\eta) \frac{\dot{\zeta}}{V_{x}}
\end{aligned}
$$

According to Eq. (12), if the effective rolling radius decreases because of tire deflation, the tire spring stiffness also decreases and the tire becomes softer. This shows that as $\delta R_{e}$ increases with tire deflation, the lower vertical resonance frequencies are encountered according to Eq.(8a). In here, it is considerable that the dependence of resonance frequency of effective rolling radius oscillations on vehicle body speed is determined with the ratio of wheel speed to vehicle speed as shown in Eq.(12a). Thus, the resonance frequency reduces, when the vehicle body speed becomes higher than wheel speed under pure rolling conditions. Also, the frequency increases, if vehicle speed is lower than wheel speed as shown in Eq.(12a).

\section{Experimental Material and Method}

In this experimental study, the effective rolling radius has been measured by means of noncontact laser height sensor as shown in Fig.(3). The sensor has 100-350 mm measurement range and $0.1 \mathrm{~mm}$ resolution. This sensor has been mounted to the wheel lug nuts via adjustable mounting collets as shown in Figs. $3 \mathrm{~b}$ and $3 \mathrm{c}$. In this way, it is located exactly in the wheel center, and it can rotate with respect to the wheel about the wheel's y-axis. To restraint this rotation, the height sensor has been connected to the vehicle body with a rod in order to assure that the sensor measures in 
the vehicle body (vertical) z-direction.

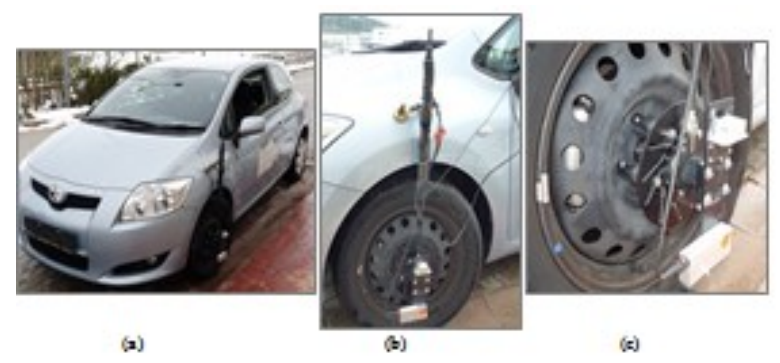

Figure 3. a) Test vehicle b) Mounting components of height sensor c) Position of height sensor on wheel

The test vehicle used for driving tests is Toyota Auris as shown in Fig. 3a. Technical specifications of test vehicle and tire are given in Table 1. After mounting this sensor onto the test vehicle, it is connected to data acquisition unit. The unit is switched according to the reference variable.

Table 1. The test vehicle specifications

\begin{tabular}{ll}
\hline \multicolumn{1}{c}{ Specifications } & \multicolumn{1}{c}{ Descriptions } \\
\hline Total vehicle weight & $1412 \mathrm{~kg}$ \\
Front axle weight & $898 \mathrm{~kg}$ \\
Rear axle weight & $514 \mathrm{~kg}$ \\
The weight acting on front tire & $449 \mathrm{~kg}$ \\
Tire dimension & $205 / 55 / \mathrm{R} 16$ \\
Tire tread depth & $7 \mathrm{~mm}$ \\
Tire nominal inflation pressure & $30 \mathrm{psi}$ \\
Tire maximum pressure & $45 \mathrm{psi}$ \\
Carcass type & $\mathrm{Radial}$ \\
Speed rating & $\mathrm{V}(240 \mathrm{~km} / \mathrm{h})$ \\
Load index & $81(462 \mathrm{~kg})$ \\
Traction & AA \\
\hline
\end{tabular}

The suspension test bench is used to determine axle and vehicle weights as shown in Fig.4. In Fig.4, the weights of the front and rear axles are firstly measured and then the total weight of vehicle is determined as shown in Table 1.
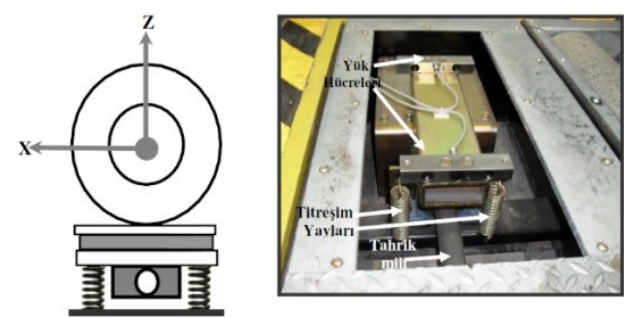

Figure 4. Measurement of axle weights

\subsection{Experimental procedure}

In this study, the reference variable is vehicle speed. Thus, the measurements are initiated and stopped within the same time period according to vehicle speed. The effects of the changes in vehicle speed on effective rolling radius are measured on dry flat road according to tire inflation pressure. Firstly, the inflation pressure of tire is set to $15 \mathrm{psi}$. Then, the test vehicle is accelerated from 0 to $90 \mathrm{~km} / \mathrm{h}$. The changes in effective rolling radius have been measured at constant vehicle speeds 30,60 and $90 \mathrm{~km} / \mathrm{h}$, respectively during acceleration maneuver. For this, when the vehicle speed reaches to $30 \mathrm{~km} / \mathrm{h}$, the vehicle speed is kept constant, and then the measurements are carried out. This measurement maneuver is repeated for 60 and $90 \mathrm{~km} / \mathrm{h}$ at same tire pressure. Also, these measurements are repeated for tire inflation pressures $20,25,30$ and $35 \mathrm{psi}$ under same road condition. As a result, all measurements are conducted under same experimental conditions for tire pressures 15, 20, 25, 30 and 35psi.

\section{Experimental Results and Discussion}

In order to investigate the effects of the changes in vehicle speed on effective rolling radius oscillations have been assessed by using Fourier Fast Transform (FFT) method as follows:

$$
X(j \omega)=\int_{-\infty}^{\infty} x(t) e^{-j \omega t} d t
$$

where $X(j \omega)$ is frequency response and $x(t)$ is effective rolling radius in time domain. Therefore, the frequency responses of effective rolling radius are shown in Fig. 5 for nominal tire pressure and lower tire pressures at 30, 60, $90 \mathrm{~km} / \mathrm{h}$. The frequency responses have two peaks at $7-20 \mathrm{~Hz}$ and $25-60 \mathrm{~Hz}$ frequency ranges. The first of these peaks shows the effective rolling radius oscillation resulting from axle resonance.

Therefore, effective rolling radius oscillations with the lowest tire pressure become the most severe at the axle resonance frequency as shown in Fig. 5a. As the vehicle speed increases, the amplitude of the oscillation decreases and the lowest amplitude is obtained at a speed of $60 \mathrm{~km} / \mathrm{h}$. In addition to, the increase in axle resonance frequencies forming a peak is remarkable when the vehicle speed increases.

On the other hand, it has been observed that the effective rolling radius oscillations are not subject to resonance at the angular frequencies of the wheel rotating with the lowest tire pressure as shown in Fig. 5a. 

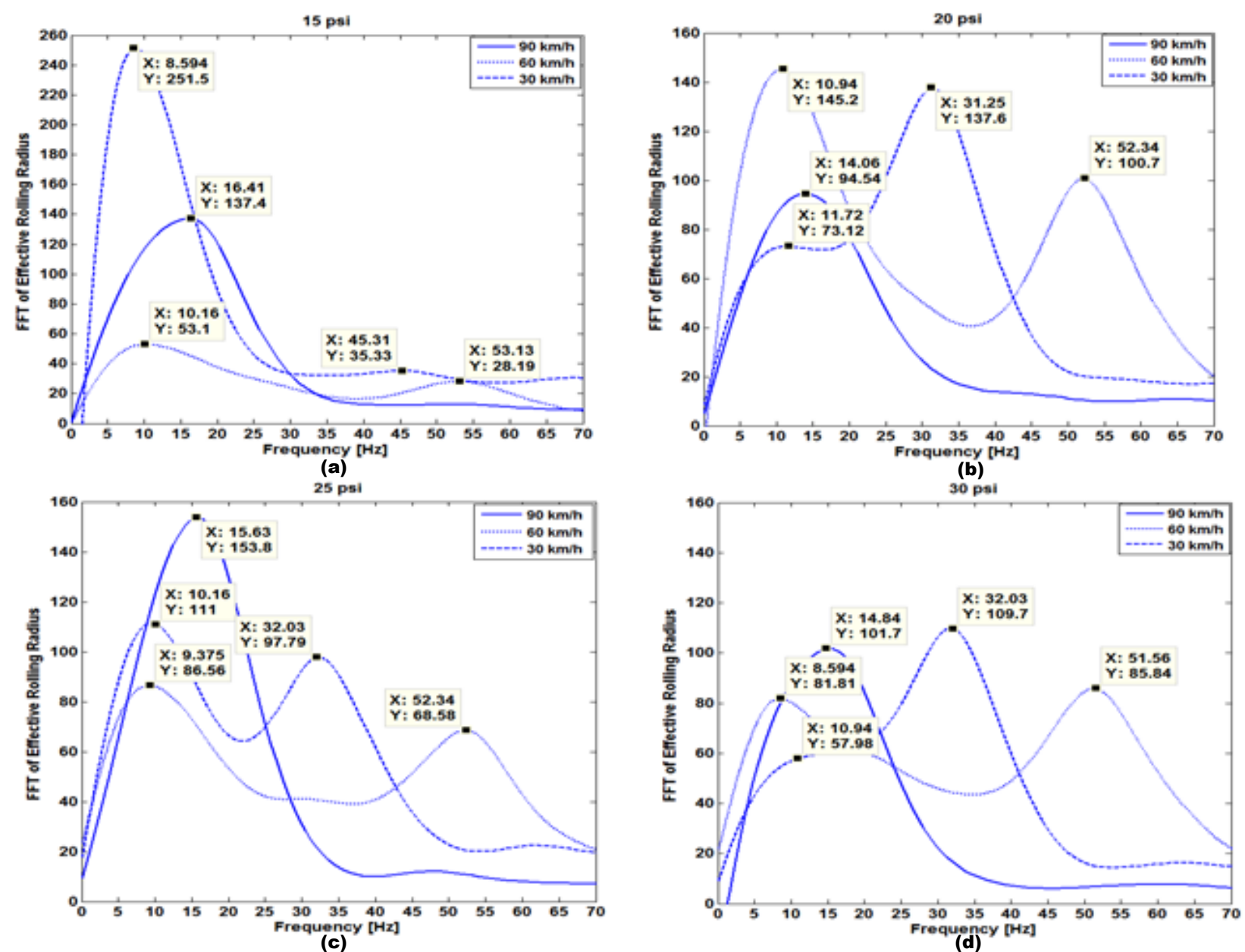

Figure 5. Frequency responses of effective rolling radius for lower tire pressures than nominal tire pressure

When the tire pressure increases by 5 psi as shown in Fig. 5b, the effect of the angular oscillations of the wheel becomes evident, whereas the amplitudes decrease considerably at axle resonance frequencies. As the vehicle speed increases, the angular resonance frequencies of both the axle and the wheel causing these amplitudes have shifted.

While the tire pressure approaches the nominal value up to 5 psi as shown in Fig.5c, the highest oscillation amplitudes are encountered at axle frequencies at $90 \mathrm{~km} / \mathrm{h}$, but the amplitudes at other speeds vary according to the tire pressure. However, at the angular frequencies of the wheel, only the amplitudes increase towards the nominal tire pressure and the resonance frequency does not shift as shown in Fig.5d.

Thus, it has been observed that the amplitudes and frequencies of the effective rolling radius oscillations differ according to the vehicle speed change in the axle resonance region. It is clear that these differences do not have a certain trend according to the increase or decrease of the tire pressure. For this reason, the change in vehicle speed makes tire pressure prediction difficult at the axle frequencies. Especially when it is desired to estimate tire pressure relative to the axle resonance frequency, the frequency increase or decrease caused by vehicle speed will cause the tire pressure change to be incorrectly determined. Also, it will be inevitable that the amplitude increases or decreases caused by the vehicle speed change will lead to false predictions when it is desired to determine the tire pressure according to the amplitude change in this frequency region.

On the other hand, it is clear that the resonance frequency is not affected by the vehicle speed change in the angular frequency region of the wheel and has the same trend as the tire pressure drops up to $10 \mathrm{psi}$. This means that the amplitudes of effective rolling radius oscillation can enable tire pressure estimation without affecting the vehicle speed in the angular frequency region of the wheel.

\section{Conclusion}

In this study, the effects of changes in vehicle speed on the changes occurring in resonance 
frequency and amplitude of effective rolling radius have been experimentally examined. For this, firstly, the tire model is established and the effects of effective rolling radius are theoretically described on the vertical resonance frequency. Then, the road tests have been conducted in constant vehicle speeds 30 , 60 and $90 \mathrm{~km} / \mathrm{h}$. Each test has been repeated for tire pressures $15,20,25$ and 30 psi. Test results are investigated by using frequency responses of effective rolling radius. The frequency responses indicate that the resonance frequency can shift at same tire pressure due to the vehicle speed changes. Thereby, TPMS systems can take wrong information about tire pressure changes because of these changes in resonance frequency resulting from vehicle speed changes at same tire pressure. Because, these systems determine tire pressure by observing the changes in frequencies that cause resonance in the effective rolling radius.

As a result, this study clearly occurs that every change in resonance frequency does not describe tire pressure changes at resonance frequency of axle. However, the amplitudes of effective rolling radius oscillation can enable tire pressure estimation without affecting the vehicle speed in the angular frequency region of the wheel, since the resonance frequency does not shift at this frequency according to vehicle speed variations. Therefore, the interaction between the resonance frequency and vehicle speed should be monitored by TPMS systems to distinguish the resonance frequency changes resulting from tire pressure changes from those of vehicle speed changes.

\section{Acknowledgements}

This study was supported by the Grants from Scientific Research Foundation of Kocaeli University (Project No. 2011/088). Also, in this project, Batuhan Polat, Metin Akyildiz, Murat Ozcu and Ayhan Senturk studied as a researcher. The author is pleased to thank the individuals and Kocaeli University who contributed to this study.

\section{References}

1. Genta, G. Motor Vehicle Dynamics: Modeling and Simulation, World Scientific Publishing, USA. Anghelache, G and Moisescu, R. (2017), The measurement of dynamic radii for passenger car tyre, IOP Conf. Ser.: Mater. Sci. Eng. 252 012014, 1997.

2. Persson, N., Ahlqvist, S., Forssell, U., Gustafsson, F. Low Tire Pressure Warning System Using Sensor Fusion," Society of Automotive Engineers Technical Paper, 200101-3337, 2001.

3. Pohl, A., Ostermayer, G., Reindl, L and Seifert, F. Monitoring the Tire Pressure at Cars Using Passive SAW Sensors. Proceedings of IEEE Ultrasonics Symposium, Toronto, 471 474, 1997.

4. Elfasakhany, A Tire Pressure Checking Framework: A Review Study, Reliability Engineering and Resilience Vol:1, No:1, 12-28, 2019.

5. Sankaranarayanan, V and Güvenç, L Tire Pressure Monitoring, IEEE Control Systems Magazine, December 2007.

6. Fileming,B Tire Pressure-Monitoring Systems Rollout, IEEE Vehicular Technology Magazine, September 2009.

7. Prashant. G.S., Harshal R. K., Saiprasad N.K., Ayaan A. K. Automatic Tyre Pressure Monitoring System Using Wireless Communication, International journal of Advance Research in Science and Engineering, Vol:3, 471-479.

8. Reina, G., Gentile, A. Messina, A. (2015) Tyre pressure monitoring using a dynamical model-based estimator, Vehicle System Dynamics, Vol. 53, No. 4, 568-586, 2017.

9. Wang, L., Zhang, Z., Yao, Y., Han, Zongqi, Wang, B., Wang, L Monitoring Method of Indirect TPMS Under Steering Situation, 3rd International Conference on Mechanical, Industrial, and Manufacturing Engineering-MIME, 2016.

10. Goharimaneshi, M., Riahi, A., Lashkaripour, A., Akbari, A. A. Tire Inflation Pressure Estimation Using Identification Techniques, International Journal of Software Engineering and Its Applications Vol. 10, No. 7, 135-144, 2016.

11. Anghelache, $G$ and Moisescu, R., The measurement of dynamic radii for passenger car tyre, IOP Conf. Ser.: Mater. Sci. Eng. 252 012014, 2017.

12. Zhu, B., Han, J., Zhao, J. Tire-Pressure Identification Using Intelligent Tire with Three-Axis Accelerometer, Sensors Vol:19, 
2560.

13. Pacejka, H.B. Tyre and Vehicle Dynamics-Second Edition, Butterworth Heinemann, United Kingdom, 2006.

14. Wong, J.Y. Theory of Ground Vehicles - Second Edition, WILEY, New York, 1993. 